\title{
PROKLA-Redaktion
}

\section{Editorial: Ökonomie der Technik}

Seit den industriellen Neuerungen der zweiten Hälfte des 18. Jahrhunderts galten technische Entwicklungen den Apologeten wie den Kritikern der entstehenden kapitalistischen Gesellschaften als Motor für Wachstum und Wohlstand. Im Kommunistischen Manifest von 1848 bescheinigten Marx und Engels der Bourgeoisie, „massenhaftere und kolossalere Produktionskräfte" geschaffen zu haben als alle früheren Generationen: „Unterjochung der Naturkräfte, Maschinerie, Anwendung der Chemie auf Industrie und Ackerbau, Dampfschiffahrt, Eisenbahnen, elektrische Telegraphen, Urbarmachung ganzer Weltteile, Schiffbarmachung der Flüsse..." (MEW 4: 467). Unter den gegenwärtigen Bedingungen des privaten Eigentums nütze all dies allerdings nur den wenigsten, während die Entfesselung der produktiven Kräfte für die große Mehrheit der Bevölkerung nichts als Elend und Unterdrückung mit sich gebracht hätten. Doch reifte damit, davon waren Marx und Engels von Grund auf überzeugt, auch die materielle Basis für eine bessere Gesellschaft heran. Rund drei Jahrzehnte später schrieb Engels in Die Entwicklung des Sozialismus von der Utopie zur Wissenschaft über die „heutigen gewaltigen Produktivkräfte“: „Aber einmal in ihrer Natur begriffen, können sie in den Händen der assoziierten Produzenten aus dämonischen Herrschern in willige Diener verwandelt werden. Es ist der Unterschied zwischen der zerstörenden Gewalt der Elektrizität im Blitze des Gewitters und der gebändigten Elektrizität des Telegraphen und des Lichtbogens, der Unterschied zwischen der Feuersbrunst und des im Dienst des Menschen wirkenden Feuers." (MEW 19: 223). Entscheidend für die Nutzbarmachung der technischen Errungenschaften der Zeit sei demnach die ,gesellschaftlich planmäßige Regelung der Produktion" (ebd.). Vertrauten die Markttheoretiker auf die segensreichen Wirkungen der Konkurrenz, die technische Entwicklungen zum Wohle aller weitertreiben und der Allgemeinheit zugänglich machen würden, so wurden hier im Gegensatz dazu grundsätzlich andere gesellschaftliche Steuerungsmechanismen eingefordert. Über alle ideologischen Gräben hinweg bestand jedoch auf beiden Seiten der Glaube an eine lineare Aufwärtsentwicklung, eine glatte Optimierung und - wie man es in der Sprache des 19. Jahrhunderts ausdrückte - eine stetige „Vervollkommnung“ der Technik.

Dieser Glaube an die Unschuld der Technik ließ sich im 20. Jahrhundert nicht mehr aufrechterhalten. Im Ersten wie im Zweiten Weltkrieg wurde Technik zum Instrument von militärischem Massenmord. Giftgas, Maschinengewehre, Raketen, Bombenflugzeuge und Panzer eröffneten neue, bisher nicht vorstellbare Schreckensdimensionen für die Soldaten wie für die Zivilbevölkerung. Zum Ende des Zweiten Weltkriegs folgten die dramatischen und traumatischen Erfahrungen der Wirkungen atomarer Energie: Die Zündung der US-amerikanischen Atombomben über Hiroshima und $\mathrm{Na}$ gasaki im August 1945 kostete damals unmittelbar und in ihren Spätwirkungen 
insgesamt mehr als 400.000 Menschen das Leben. Die ungeheure Zerstörungskraft des „Feuerregens“ veranlasste den Philosophen Günther Anders zu der pessimistischen Diagnose der „Antiquiertheit des Menschen“, der mit den von ihm selbst entwickelten technischen Potentialen offenbar nicht Schritt halten konnte und die Kontrolle über deren vernünftigen Gebrauch endgültig verloren hatte. Doch das apokalyptische Grauen, das die Bombenabwürfe verbreiteten, wurde durch das nachfolgende Versprechen einer „friedlichen Nutzung“ bald in den Hintergrund gedrängt. In der Bundesrepublik wurde 1955 unter FranzJosef Strauß ein eigenes Ministerium für Atomfragen eingerichtet und Strauß erklärte die Entwicklung der Atomenergie zur nationalen „Existenzfrage“. Die Stromkonzerne standen der neuen Technologie zunächst äußerst skeptisch gegenüber, wurden dann jedoch mit Hilfe von Milliardengeldern für die Subventionierung der Forschung sowie durch günstige Kredite, Verlustgarantien und Bundesbürgschaften umgestimmt. Aber auch linke Theoretiker wie Ernest Mandel oder Ernst Bloch gaben sich dem Traum vom Goldenen Atomzeitalter mit unbegrenzten Energieressourcen, nukleargetriebenen Autos und Flugzeugen hin. Bloch schwärmte in Das Prinzip Hoffnung vom grandiosen Nutzen der Kernkraft: „Wie die Kettenreaktionen auf der Sonne uns Wärme, Licht und Leben bringen, so schafft die Atomenergie, in anderer Maschinerie als der der Bombe, in der blauen Atmosphäre des Friedens aus Wüsten Fruchtland, aus Eis Frühling. Einige hundert Pfund Uranium und Thorium werden ausreichen, um die Sahara und die Wüste Gobi verschwinden zu lassen, Sibirien und Nordamerika, Grönland und die Antarktis zur Riviera zu verwandeln." (Das Prinzip Hoffnung, Bd. 2, Ffm. 1959, S. 775). Erst im Gefolge der Studentenbewegung formierte sich eine kritische Öffentlichkeit, die in den 1970er Jahren die unabsehbaren Sicherheitsrisiken der zivilen wie der militäri- schen Nutzung der Kernenergie in Ost und West sowie die bis heute völlig ungelöste Frage der Endlagerung von abgebrannten Brennstäben aufwarf.

Nachdem die Gefahren der Atomtechnologie in Deutschland und anderswo durch Bürgerbewegungen und kritische WissenschaftlerInnen bekannt gemacht worden waren, konnten sie nicht mehr ignoriert werden. Dazu kamen seit Ende der 1950er Jahre eine Reihe von schweren Störfällen - 1957: Windscale/Sellafield, 1957/58: Tscheljabinsk, 1979: Harrisburg, 1986: Tschernobyl, 1999: Tokaimura. Wie wenig beherrschbar die Atomtechnologie trotz gestiegener Sicherheitsstandards nach wie vor geblieben ist, zeigten immer wieder zahllose kleinere Störfälle, in jüngster Zeit unter anderem in Temelin und Forsmark. Doch das Entsetzen über Tschernobyl ist für viele in eine entfernte Vergangenheit gerückt und so stoßen die Pläne vieler Regierungen, weltweit neue Reaktoren zu bauen oder, wie in Deutschland, den „Ausstieg aus dem Ausstieg“ ins Auge zu fassen, nur auf mäßigen Widerstand. Vielmehr scheint die frühere verhängnisvolle Aufspaltung der Gefahren in eine bedrohliche militärische und in eine vorgeblich harmlose zivile Variante wiederaufzuleben: Im Jahr 2005 wurde der Friedensnobelpreis an die Internationale Atomenergiebehörde (IAEA) unter ihrem Direktor Mohamed El Baradei verliehen: für ihre „Bemühungen, die Nutzung der Atomenergie für militärische Zwecke zu verhindern und ihre friedliche Nutzung so sicher wie möglich zu gestalten." Allein in Europa sollen nach Angaben von Greenpeace in den nächsten Jahren 29 neue Reaktoren gebaut werden - Pläne, die für ungleich weniger Aufregung sorgen als die Behauptung der nordkoreanischen Regierung, über Atomraketen zu verfügen.

Im Anschluss an die Sensibilisierung der Öffentlichkeit für die Unwägbarkeiten und Risiken der Atomtechnologie entstand eine breite sozialwissenschaftliche Diskussion über „angepasste“ Technolo- 
gien - wie in entwickelten und auch in weniger entwickelten Ländern Techniken entworfen und eingesetzt werden sollten, die gleichermaßen sozial und ökologisch verträglich wären. Die Kritik an Großtechniken hatte sich nicht nur gegen Atomkraftwerke, sondern auch gegen die Chemieindustrie, die industrialisierte Landwirtschaft sowie die Luft- und Raumfahrttechnik gerichtet. In dieser Situation trafen „Kleinheitstheoretiker“ wie Leopold Kohr und Ernst F. Schumacher auf offene Ohren. Heute wird ihr Konzept „Small is beautiful“ meist nur noch verkürzt als Kritik an Großtechnologien gesehen. Doch diese richtete sich ebensosehr gegen den Imperativ des ständigen Wachstums und wies, wie auch andere Schriften dieser Zeit, deutlich auf mögliche Grenzen des Wachstums hin. Diese grundsätzliche Kritik ist mittlerweile in weiten Kreisen verloren gegangen. Übrig geblieben ist eine Suche nach Technologien, die mehr Dinge mit weniger Naturverbrauch produzieren sollen. Ressourceneffiziente Technik soll nun besseres Wachstum ermöglichen.

Die Frage, wie technische Entwicklung, wirtschaftliche Interessen, politische Rahmenbedingungen und Herrschaftsverhältnisse generell zusammenhängen, wird seit den 1990er Jahren kaum noch gestellt. Technikfolgenabschätzung ist zwar auf verschiedenen Ebenen etabliert worden, hängt jedoch direkt von staatlichen Mitteln $\mathrm{ab}$ und ist somit nur gefragt und förderungswürdig, soweit sie darzulegen weiß, wie sie einen Beitrag zur „Innovationsfähigkeit" leisten kann. Damit sind die hochgespannten Erwartungen auf bessere Partizipationsmöglichkeiten durch Technikfolgenabschätzung verschwunden und wurden mittlerweile von Staats wegen durch den affirmativen Begriff der Innovations- und Technikanalysen (ITA) ersetzt. Während die internationalen und interdisziplinären Science and Technology Studies (STS) seit den achtziger Jahren die soziale und kulturelle Konstruiertheit von Wissen und Technologien analysiert haben, blieb die ökonomische Dimensi- on der Technik, mit Ausnahme einiger Mikrostudien, eine Leerstelle. Demgegenüber soll in diesem Heft der PROKLA wieder an „traditionelle“ Fragestellungen angeknüpft werden, bei denen es um das Wechselverhältnis technischer und wirtschaftlicher Entwicklungen und Interessen geht oder auch um gesellschaftliche Heilsversprechen, die mit Innovationen und dem Einsatz neuer Techniken verbunden sind. Lässt man die, nach den Erfahrungen des 20. Jahrhunderts naiv erscheinende Fortschrittsgläubigkeit früherer Theoretiker hinter sich, so lassen sich mehrere Ebenen der Betrachtung unterscheiden.

Auf einer ersten Ebene geht es um die Widersprüche zwischen technischer und ökonomischer Rationalität. Sozialdemokraten, die sich spätestens nach dem Ersten Weltkrieg von der Vision einer grundlegenden Umgestaltung der Gesellschaft verabschiedet und mit den ökonomischen Verhältnissen arrangiert hatten, stimmten in den Chor der bürgerlichen Fortschrittsbefürworter ein und feierten Henry Fords Popularisierung des Autos mit Hilfe von Spezialmaschinen, Fließbändern und dem Einsatz von angelernten Arbeitern. Ähnlich gingen die meisten Sozialwissenschaftler der 1920er Jahre, etwa Friedrich von Gottl-Ottlilienfeld, von einer inhärenten „technischen Vernunft" aus. Wie Roman Köster in seinem Beitrag zu den Technikkonzeptionen der damaligen nationalökonomischen Diskussionen aufzeigt, musste sich die Hoffnung auf schrankenlose Technisierung der Produktion jedoch bald an der „ökonomischen Vernunft “ brechen die Logik des Kapitals stand der Ausschöpfung aller technischen Potentiale oftmals entgegen oder aber sie produzierte Überkapazitäten und Arbeitslosigkeit. Vielfach wurde dem Staat oder dem Konzept der „gebundenen Wirtschaft" daher zugetraut, hier eine neue gesellschaftliche Harmonie ins Werk zu setzen.

In den Jahrzehnten nach dem Zweiten Weltkrieg zeigte sich in der Bundesrepublik immer wieder, dass technische In- 
novationen vielfach an den sozio-ökonomischen Rahmenbedingungen scheiterten: etwa aufgrund der Konkurrenzsituation oder etablierter Technologien, die eine hohe Pfadabhängigkeit aufweisen und der Einführung neuer Technologien entgegenstanden. Entgegen den vorherrschenden Ansichten sind erfolgreiche Innovationen nicht einfach durch überlegenes Management und vermeintlich objektive technische Überlegenheiten „herstellbar“", sondern hochgradig von bestehenden Macht- und Marktverhältnissen abhängig. Bekannte Beispiele für „Flops“, für gescheiterte Innovationen der letzten Jahrzehnte sind das Plastikfahrrad Itera, der Schnelle Brüter, das Riesenwindrad Growian oder das RiesenLuftschiff Cargolifter. Reinhold Bauer stellt einige Fälle, insbesondere den Hydrobergbau und den Sterling-Motor, vor. Eine zweite Ebene möglicher Konflikte ergibt sich aus beabsichtigten oder unbeabsichtigten Wirkungen der technischen Entwicklung, die einzelne Brancheninteressen unterschiedlich berühren können. Diese Entwicklung folgt keinem Masterplan, sondern den individuellen Kalkülen einzelner Kapitale, womit Substitutions- und Verdrängungsprozesse ausgelöst werden können (etwa des Gaslichts durch elektrische Beleuchtung, der Schallplatte durch die Compact Disc). Oder aber die Abnehmer bekommen technische Geräte zur Verfügung, deren Nutzung sich unmittelbar gegen die Interessen anderer Unternehmen richtet dies ist in den letzten Jahren durch Techniken zum Kopieren und Reproduzieren von Musikaufnahmen oder Filmen der Fall. Lars Bretthauer untersucht, wie sich die Digitalisierung von Filmen auf die Filmindustrie ausgewirkt hat und wie diese unter anderem versucht, staatliche Instanzen einzuschalten, um der weitverbreiteten, in ihren Augen jedoch äußerst schädlichen Praxis des downloads und der unentgeltlichen Weitergabe von Filmen beizukommen.

Eine dritte, seit den Kontroversen um die Atomtechnologie vieldiskutierte
Ebene berührt die Frage, inwieweit technische Entwicklungen demokratisch kontrollierbar sind und inwieweit sie im Interesse der NutzerInnen - der Konsumentlinnen, der abhängig Beschäftigten, der Gesellschaft insgesamt - gestaltet werden können. In den 1970er Jahren wurden die bereits erwähnten Verfahren der Technikfolgenabschätzung entwikkelt, mit denen entsprechende Hoffnungen umgesetzt werden sollten. Technikhistorikerlinnen meinten, aus Studien zur Technikgenese früherer Neuerungen ließen sich Folgerungen ziehen, die auch prospektiv verwendet werden könnten. Doch diese optimistischen Erwartungen ließen sich allenfalls punktuell umsetzen, unter anderem deshalb, weil zukünftige Entwicklungen häufig nicht den Mustern der Vergangenheit folgen, sondern immer wieder neue Wege eingeschlagen werden. Aber auch das wirtschaftliche und politische Umfeld änderte sich mit der zunehmenden Internationalisierung der Märkte, so dass die staatliche Einflussnahme der 1970er Jahre unter anderen Vorzeichen stand als diejenige am Ende des 20. Jahrhunderts. Petra Schaper-Rinkel nimmt in den Blick, wie die Nanotechnologie als Schlüsseltechnologie des 21. Jahrhunderts politisch formiert wird und dabei eine Ökonomie des Versprechens etabliert wird.

Eine vierte Ebene der Betrachtung führt zu den eingangs angeführten Hoffnungen zurück, neue technische Entwicklungen führten unmittelbar zu besseren Lebensverhältnissen. In der sozialwissenschaftlichen Forschung gilt eine derartige Vorstellung als Technikdeterminismus und als lange überwunden. Wenngleich bestimmte Technologien wie die Kernenergie ihr Gefahrenpotential unter unterschiedlichen gesellschaftlichen Bedingungen unverändert beibehalten, erweist sich in den meisten anderen Fällen der soziale Kontext des Einsatzes als entscheidend. Diese Einsicht wurde unter anderem bei der Untersuchung von Produktionsprozessen und deren Technisierung gewonnen. Häufig schreiben Ma- 
schinen und Arbeitsmittel keineswegs zwangsläufig eine bestimmte Arbeitsteilung vor - dies war zwar der Fall bei Henry Fords Spezialmaschinen und Fließbändern, die für angelernte Arbeitskräfte vorgesehen waren und ein bestimmtes Arbeitstempo vorgaben, nicht aber bei der Einführung der NCWerkzeugmaschine - um dieses Beispiel stellvertretend für viele andere anzuführen. Wie David Noble gezeigt hat, konnte ihr Einsatz ebenso damit verbunden sein, dass Kopf- und Handarbeit (Programmierung und Bedienung) auf zwei Arbeitskräfte aufgeteilt wie auch bei einer Arbeitskraft zusammengeführt waren. Um so mehr gilt diese Kontextabhängigkeit im Bereich des Konsums. Wie Handys, Tiefkühlkost, Autos oder VideoRecorder genutzt und benutzt werden, hängt von spezifischen Kulturen $a b$, die sich unter anderem nach Ländern und Regionen sowie nach Geschlecht und Alter unterscheiden. Im Widerspruch $\mathrm{zu}$ diesen Erkenntnissen ist die Vorstellung, Technik gestalte unmittelbar Verhältnisse, in weiten Teilen der Öffentlichkeit wie der Politik immer noch vorherrschend. So stellt die Wirtschaftswoche etwa das „Krankenhaus der Zukunft“ vor, in dem aus einfachen Patienten dank Computertechnik „digitale Patienten“ werden. Die „totale Vernetzung von Arzt und Patient", wird in dem Artikel mit Emphase behauptet, „spart Kosten und kann Leben retten." (Wirtschaftswoche Nr. 30/ 2006: 77ff). Auch in anderen Bereichen wird die Komplexität sozialer und kultureller Kontexte häufig äußerst vereinfacht und unzulässig verkürzt. Karsten Weber unterzieht daher Projekte, mit denen der Digital Divide, also die Kluft zwischen Besitzern und Nicht-Besitzern von Computern in entwickelten und weniger entwickelten Ländern überbrückt werden sollte, einer kritischen Überprüfung.

Ein klassisches Gebiet für technikdeterministische Sichtweisen stellt schließlich von jeher das Militär dar. Die Entwicklung einer „Wunderwaffe“ galt nicht nur der Deutschen Wehrmacht im Zweiten
Weltkrieg als bevorzugtes Mittel, um die gegnerischen Alliierten endgültig zu besiegen. Ähnlich wie hinter dem „digitalen Patienten“ ein grundlegend neues $\mathrm{Pa}$ tientenverständnis steht, hat das USMilitär in den letzten Jahren einen „digitalen Soldaten“ konstruiert, der auf einem „gläsernen Schlachtfeld" agieren und mit dem großen Versprechen einer „risikolosen Kriegsführung“ (dies allerdings nur bezogen auf die US-Soldaten) antreten würde. Jan Benedix befasst sich mit der Frage, welche Rolle derartige Leitbilder für die neuen Konzeptionen der US-Streitkräfte spielen.

Technikdeterminismus erlebt demnach in der medialen, wirtschaftlichen und politischen Praxis eine anhaltende Konjunktur, bietet er sich doch nach wie vor für einfache und leicht vermittelbare Weltbilder und -erklärungen an. Als einer der Väter solcher Sichtweisen gilt Marx. „Die Handmühle ergibt eine Gesellschaft mit Feudalherren, die Dampfmühle eine Gesellschaft mit industriellen Kapitalisten“, schrieb er 1847 in der Schrift Elend der Philosophie (MEW 4: 130). Diese pointierte Formulierung brachte ihm den Vorwurf ein, trotz aller Betonung von sozialen Auseinandersetzungen und Klassenkämpfen im Grunde Technikdeterminist zu sein, die Technik also zur zentralen Akteurin gesellschaftlicher Veränderungen zu machen. So sehr Marx aber, wie eingangs angemerkt, die technischen Entwicklungen seiner Zeit hervorhob und offen oder verstohlen bewunderte, so war ihm eine solche Sichtweise dennoch - wie allein ein Blick in den ersten Band des Kapitals zeigt zutiefst fremd. Nicht Maschinen machten ihm zufolge Geschichte, sondern Menschen innerhalb bestimmter gesellschaftlicher Bedingungen. Dazu gehörten auch die jeweiligen technologischen Standards einer Zeit, die ihrerseits durch wirtschaftliche Aktivitäten verändert wurden. Die zentrale Rolle spielten dabei die kapitalistischen Unternehmer, die technische Neuerungen wie die Spinning Jenny von James Hargreaves oder den 
mechanischen Webstuhl von Edmund Cartwright im Wesentlichen aus zwei Gründen einsetzten: um die Produktionskosten zu senken und in dem Bestreben, die fallweise aufsässige Arbeiterschaft zu disziplinieren. Anders als viele vereinfachende Deutungen seiner Epigonen analysierte Marx den Prozess der Einführung neuer Arbeitsmethoden und der Mechanisierung der Arbeit jedoch höchst differenziert und widmete sich auch den inneren Widersprüchlichkeiten dieser Prozesse. Einer der am häufigsten zitierten Sätze aus dem „Maschinerieka-

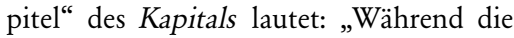
Maschinenarbeit das Nervensystem aufs äußerste angreift, unterdrückt sie das vielseitige Spiel der Muskeln und konfisziert alle freie körperliche und geistige Tätigkeit. Selbst die Erleichterung der Arbeit wird zum Mittel der Tortur, indem die Maschine nicht den Arbeiter von der Arbeit befreit, sondern seine Arbeit vom Inhalt." (MEW 23: 446) Doch die detailreiche Schilderung dieser Tendenz endet in einer überraschenden Wendung, mit der die vorhergehenden Ausführungen scheinbar auf den Kopf gestellt werden: „Die moderne Industrie betrachtet und behandelt die vorhandne Form eines Produktionsprozesses nie als definitiv. Ihre technische Basis ist revolutionär, während die aller früheren Produktionsweisen wesentlich konservativ war... Sie revolutioniert damit ebenso beständig die Teilung der Arbeit im Innern der Gesellschaft... Die Natur der großen Industrie bedingt daher Wechsel der Arbeit, Fluss der Funktion, allseitige Beweglichkeit des Arbeiters." (MEW 23: 510f). Tatsächlich zeigte sich im folgenden Jahrhundert ein Nebeneinander unterschiedlichster Methoden, um die Arbeitsproduktivität zu steigern. „Reelle Subsumtion" konnte mit zunehmender Spezialisierung und unmittelbarer Kontrolle der Tätigkeiten, aber auch mit der Förderung von Facharbeit und relativ autonomen Formen der Arbeitsorganisation einhergehen. Im Gegensatz zu dem idealisierenden Blick, den Marx auf die
Entwicklung von Technik als fraglose Grundlage von Wohlstand und gutem Leben warf, ist von seinen Überlegungen zum konkreten Einsatz von Technik an Arbeitsplätzen immer noch zu lernen. Auch in anderen Bereichen bleibt Technik nach wie vor im Rahmen kapitalistischer Produktions- und Verwertungsverhältnisse und ihrer Widersprüche zu thematisieren.

Der Computerentwickler und -Kritiker Joseph Weizenbaum hat die Computertechnik die Technik genannt, die in der zweiten Hälfte des 20. Jahrhunderts Eigentums- und Herrschaftsstrukturen befestigt hat: „Ja, der Computer kam 'gerade noch rechtzeitig'. Aber rechtzeitig wofür? Er kam gerade noch rechtzeitig, um gesellschaftliche und politische Strukturen intakt zu halten - sie sogar noch abzuschotten und zu stabilisieren -, die andernfalls entweder radikal erneuert worden oder unter den Forderungen ins Wanken geraten wären, die man unweigerlich an sie gestellt hätte." (Die Macht der Computer und die Ohnmacht der Vernunft, Ffm. 1978, S. 54). Ob sich Ähnliches noch einmal ereignen könnte, steht heute mit der Umwelttechnik auf der politischen Tagesordnung. Nachdem neuerdings sogar europäische Regierungschefs wie Tony Blair von der kommenden Klimakatastrophe sprechen, soll Umwelttechnik als Rettungsanker für das fossile industrielle System dienen, dabei auch noch neue Absatzmärkte für die Länder schaffen, die den Klimakollaps maßgeblich $\mathrm{zu}$ verantworten haben. Erneut ist das Ziel die Quadratur des Kreises: Umwelttechnologien, so die Europäische Kommission, „führen zu einer Senkung des Energie- und Ressourcenverbrauchs und damit zur Verbesserung der Wettbewerbsfähigkeit bei gleichzeitiger Verringerung von Emissionen und Abfällen" (Mitteilung an den Rat und das Europäische Parlament: Stimulation von Technologien für nachhaltige Entwicklung KOM 2004/38). Seit auch die herrschende Politik festgestellt hat, dass die Weiter-so-Strategie keine langfristig mög- 
liche energiepolitische Perspektive sein kann, lautet die Losung der deutschen Umweltpolitik, deutsche Unternehmen zu Marktführern auf dem wachsenden Weltmarkt für Umwelttechnologien zu machen. Da jedoch die Frage der Ressourcen nur die sichtbare Spitze des Eisbergs bildet, können technische Lösungen zur Steigerung der Energie- und Res- sourceneffizienz lediglich die Symptome eindämmen. Das generelle Problem, das vom kapitalistischem Wachstum ausgeht, wird mit den technischen Lösungen zur Effizienzsteigerung aber nicht gelöst. Nicht allein technische, sondern grundlegende politische und ökonomische Veränderungen sind hier nötig.

PROKLA 146: „... der Bevölkerung“ - Kritik der Demographie (März 2007) PROKLA 146 nimmt die Renaissance der Thematik „Demographie“ zum Anlass für eine kritische Analyse der damit veranstalteten „reformpolitischen“ Mobilmachung. Das Heft fragt zum einen nach den am Prozess des demografischen Wandels und seiner politischen Bearbeitung deutlich werdenden Grenzen hergebrachter politischer und sozialer Vergemeinschaftung. Zum anderen wird das von Wissenschaft und Feuilleton gezeichnete Schreckensbild einer Gerontokratie, in der die Alten der Zukunft die Tyrannei ausüben, als auch die angebotenen Lösungsmöglichkeiten, durchleuchtet.

\section{PROKLA 147: Internationalisierung des Staates (Juni 2007)}

Im Zuge der Globalisierung haben sich sowohl die Voraussetzungen, unter denen Politik stattfindet, als auch die Rahmenbedingungen politischer Auseinandersetzungen und Kämpfe erheblich geändert. Gleichzeitig wird durch diese Kämpfe aber auch das Terrain des „Internationalen Politischen“ mit konstituiert. Die Veränderungen, die die Medien staatlicher Herrschaft - Geld, Macht und Recht auf internationaler Ebene erleben, will PROKLA 147 untersuchen, ebenso wie den Zusammenhang zwischen transnationaler Klassenbildung und der Internationalisierung von Staatlichkeit sowie den Möglichkeiten, die sich für eine herrschaftskritische Politik unter diesen Bedingungen ergeben. 